\title{
Optimal reactive power planning considering the adjustment coefficient of generator excitation system
}

\author{
Bailin Liu ${ }^{1}$ Xingwei $\mathrm{Xu}^{2}$ \\ ${ }^{1}$ School of Electrical \& Electronic Engineering, North China Electric Power University, Beijing \\ 102206, China \\ ${ }^{2}$ State Grid Corporation of China, Northeast Division, Shenyang 110181, China
}

Email: liubailin0424@163.com

\begin{abstract}
The generator excitation system adjustment coefficient determines the reactive power control features of the generator. Reasonable setting of the excitation system adjustment coefficient can improve the reactive power support capacity of the generator to the regional power grid. In this paper, we propose a reactive power optimal planning model, which takes into account the generator excitation system adjustment coefficient, fully exploit the reactive power voltage control capacity of the generator in the reactive power optimal planning and utilize the Benders decomposition algorithm to work out an optimized solution. Results of grid simulation show that the proposed method can improve the grid voltage level and reduce the comprehensive operating costs of the power grid.
\end{abstract}

Keywords: Reactive Power Optimal Planning, Excitation System Adjustment Coefficient, Benders Decomposition.

\section{INTRODUCTION}

With the rapid economic growth, the electric power load is increasing, and the power grid structure is becoming more and more complicated, raising higher requirements for the reliability of power supply. Therefore, it is very important to carry out reasonable configuration of the system reactive power source and reactive power compensation devices to ensure the safe and economic operation of the power grid [1].

The generator is an important reactive power supply of the power grid. The excitation system adjustment coefficient refers to the relationship between the change in the generator terminal voltage and the change in the reactive power of the generator, which determines the reactive voltage control features of the generator. The reactive power adjustment of generators has a large range and needs no additional investment [2-4]. Reasonable setting of the generator excitation system adjustment coefficient can improve the grid voltage level, reduce power grid losses [5-6] and improve the stability of power grid operation [7-11].

Reactive power optimal planning is part of the power system optimal planning. It means reasonably adjusting the reactive power of the generator and determining the installed locations and optimal capacity of the reactive power compensation device within the planned period while ensuring the safe operation of the system to improve the grid voltage level, reduce active power loss and minimize the sum of the power loss costs and equipment investment costs.
Researchers from home and abroad have studied the reactive power optimal planning. Reference [12-13] studied the reactive power optimal planning methods for power grids with stable voltages. Reference [14-15] discussed the reactive power optimal planning method considering multiple operation modes. The current researches on reactive power optimal planning do not consider how different setting values of the generator excitation system adjustment coefficient affect the reactive power optimal planning results

In this paper, we propose a regional grid reactive power optimal planning model which considers the generator excitation system adjustment coefficient, carry out the calculation, take the combined action of the set values of the generator excitation system adjustment coefficient and the reactive power compensation device into overall account, and apply the model in the actual power grid. The results show that while saving equipment investments, this method can also further improve the grid voltage level and reduce grid loss, which is of great significance to the reactive power optimal allocation of the regional power grid.

\section{THE GENERATOR EXCITATION SYSTEM ADJUSTMENT COEFFICIENT}

The generator excitation system adjustment coefficient studied in this paper refers to the relationship between the busbar voltage on the high voltage side of the generatortransformer set and the reactive power of the generator, i.e.: 
$\beta=-\frac{V_{r e f}-V_{G}}{Q_{r e f}-Q_{G}}$

where $V_{\text {ref }}$ is the target value of the voltage on the high voltage side; $Q_{\text {ref }}$ is the reference value of the generator reactive power and $Q_{G}$ is the generator reactive power.

\section{REACTIVE POWER OPTIMAL PLANNING MODEL CONSIDERING THE GENERATOR EXCITATION SYSTEM ADJUSTMENT COEFFICIENT}

\subsection{Objective function}

Based on the grid planning and load forecasting results, we obtain the data of multiple target operating modes in the planned target year and establish the target function as shown in (2) with the generator excitation system adjustment coefficient and the reactive power compensation capacity as the control variables, the voltage and generator reactive power as constraints, and minimizing the sum of the power loss costs and reactive power compensation device investments as the objective:

$$
\min \left(C_{0} Q_{c}+\sum_{1}^{k} C_{i}\left(\beta, Q_{c}\right)\right)
$$

where, $\beta$ is the value of the excitation system adjustment coefficient of the generator set involved in the optimal setting. $Q_{c}$ is the newly increased capacity of the reactive power compensation device, $C_{0} Q_{c}$ is the investment cost of the reactive power compensation device and $\sum_{1}^{k} C_{i}\left(\beta, Q_{c}\right)$ is the sum total of the grid loss costs under k operation modes.

\subsection{Constraints}

As reactive power is locally balanced, there is usually no large-scale reactive power transmission between regional power grids. Therefore, in this paper, we only consider the optimal planning calculation when the reactive load is inductive and only discuss the investments in the capacitors.

From Formula (1), we can obtain the power flow calculation models considering the generator excitation system adjustment coefficient and reactive power compensation capacity.

Constraints under each operation mode are:

(1) Flow constraints

$$
\left\{\begin{array}{c}
P_{i}-V_{i} \sum_{j \in i} V_{j}\left(G_{i j} \cos \theta_{i j}+B_{i j} \sin \theta_{i j}\right)=0 \\
i=1, \cdots, n-1 \\
\left(\frac{V_{i r e f}-V_{i}}{\beta_{i}}+Q_{\text {Giref }}\right)- \\
V_{i} \sum_{j \in i} V_{j}\left(G_{i j} \sin \theta_{i j}-B_{i j} \cos \theta_{i j}\right)=0 \\
i=1, \cdots, r \\
\left(Q_{i}+Q_{C i}\right)-V_{i} \sum_{j \in i} V_{j}\left(G_{i j} \sin \theta_{i j}-B_{i j} \cos \theta_{i j}\right)=0 \\
i=1, \cdots, s \\
Q_{L i}-V_{i} \sum_{j \in i} V_{j}\left(G_{i j} \sin \theta_{i j}-B_{i j} \cos \theta_{i j}\right)=0 \\
i=1, \cdots, n-m-r-s-1
\end{array}\right.
$$

where, $\mathrm{n}$ is the total number of nodes, $\mathrm{m}$ is the number of PV nodes, $\mathrm{r}$ is the number of $P \beta$ nodes, $\mathrm{s}$ PQ nodes are with the reactive power compensation devices and n-m-r-s- 1 nodes are without the reactive power compensation devices.

(2) Control variable constraints

The control variables are the generator excitation system adjustment coefficient and the capacity of $s$ reactive power compensation devices. The constraint for the generator excitation system adjustment coefficient is:

$$
\beta_{\text {min }} \leq \beta_{i} \leq \beta_{\text {max }}
$$

The constraint for the reactive power compensation capacity is:

$$
Q_{c} \leq Q_{c \max }
$$

\section{(3) Safety constraints}

The constraint for the voltage operation at each node of the grid is:

$V_{i \min } \leq V_{i} \leq V_{i \max }$

The constraint for the reactive power operation of the generator is:

$Q_{G i \min } \leq Q_{G i} \leq Q_{G i \max }$

\section{REACTIVE POWER OPTIMAL PLANNING METHOD CONSIDERING THE GENERATOR EXCITATION SYSTEM ADJUSTMENT COEFFICIENT}

In the generator excitation system adjustment coefficient optimization model for grid planning, the control variables include the capacity of the reactive power compensation device and the values of generator excitation system adjustment coefficient, but the installed locations and capacities of the reactive power compensation devices are unknown, making it difficult to solve the objective function. Therefore, we divide the solving process into two parts:

Step 1: under various operation modes, we use the Benders decomposition algorithm to break down Formula (2) into the operation subproblem and the investment subproblem. By iteration of the operation subproblem and the investment subproblem, we obtain the installed location and capacity of the reactive power compensation device under each operation mode, and at last calculate the capacity of each reactive compensation node as the maximum capacity under all operation modes;

Step 2: where the installed location and capacity of the reactive power compensation device are known, for the set value of the excitation system adjustment coefficient of any generator set, we can obtain the sum of the grid losses under all operation modes through load flow calculation. By adding investment costs and grid loss costs, we can obtain the objective function value of the adjustment coefficient. And by utilizing the improved particle swarm optimization algorithm, we can obtain the optimized result of the generator excitation system adjustment coefficient. 


\subsection{Calculation of the installed location and capacity of the reactive power compensation device}

For maximum operation mode, the optimization problem is converted to the single-mode reactive power optimal planning problem. To solve this problem, we can use the Benders decomposition method.

The Benders decomposition method was first proposed by J.F.Benders in 1962 to solve the mixed integer programming problem. As it converts the original problem to the operation subproblem and auxiliary problems, this method is widely applied in solving the reactive power optimization for power grids [16-18].

Detailed application steps are as follows:

(1) Fix the capacity of each reactive power compensation device $Q c$, and construction the operation subproblem.

Calculate the jacobian matrix according to the power flow equation, as follows:

$$
\left[\begin{array}{c}
\Delta P \\
\Delta Q_{l} \\
\Delta Q_{c} \\
\Delta \beta
\end{array}\right]=\left[\begin{array}{cc}
H & N \\
M_{l} & L_{l} \\
M_{c} & L_{c} \\
D & W
\end{array}\right] \times\left[\begin{array}{c}
\Delta \theta \\
\Delta V
\end{array}\right]
$$

Where, $\Delta Q l$ is the reactive power increment at the node without a reactive power compensation device, $\Delta Q c$ is the reactive power increment at the node with the reactive power compensation device, and $\Delta \beta$ is the increment of the generator excitation system adjustment coefficient. Hij, Nij, $\operatorname{Mlij}(M c i j)$ and $L c i j(L c i j)$ can be obtained through the load flow calculation of the elements in the jacobian matrix under the regular polar coordinate approach. The calculation formulas for various elements in the matrix $\mathrm{D}$ and $\mathrm{W}$ are as follows:

$D_{i j}-\frac{V_{r}-V_{i}}{\left(Q_{i}-Q_{r e f}\right)^{2}} \times V_{i} V_{j}\left(G_{i j} \cos \theta_{i j}+B_{i j} \sin \theta_{i j}\right) \quad i \neq j$

$D_{i j}=\frac{V_{r}-V_{i}}{\left(Q_{i}-Q_{r e f}\right)^{2}} \times\left(P_{i}-V_{i}^{2} G_{i i}\right), i=j$

$W_{i j}=\frac{V_{r}-V_{i}}{\left(Q_{i}-Q_{r e f}\right)^{2}} \times V_{i} V_{j}\left(G_{i j} \sin \theta_{i j}-B_{i j} \cos \theta_{i j}\right) \quad i \neq j$

$W_{i j}=\frac{V_{r}-V_{i}}{\left(Q_{i}-Q_{r e f}\right)^{2}} \times\left(Q_{i}-V_{i}^{2} B_{i i}\right), i=j$

In the optimization process, we assume the active power has undergone economic dispatch, so the active power change of the balance nodes is the grid loss change, and thus,

$\Delta P_{s}=J_{s 1} \times \Delta \theta_{j}+J_{s 2} \times \Delta V_{j}$

Where, $J_{s 1}$ and $J_{s 2}$ are obtained from (14) and (15).

$$
\begin{aligned}
& J_{s 1}=-V_{s} V_{j}\left(G_{s j} \sin \theta_{s j}-B_{s j} \cos \theta_{s j}\right), i \neq j \\
& J_{s 2}=-V_{s} V_{j}\left(G_{s j} \cos \theta_{s j}+B_{s j} \sin \theta_{s j}\right), i \neq j
\end{aligned}
$$

For nodes without the reactive compensation, $\Delta Q l=0$. For nodes with the reactive compensation, $\Delta Q c=0$. By substituting Formula (13), we can obtain the mathematic model for the linear operation subproblem, which is as follows:

$$
\begin{gathered}
\min \Delta C_{0}(\Delta \beta, \Delta V)=\varepsilon\left(J_{s 1} \times K \times \Delta \beta+J_{s 2} \times \Delta V\right) \\
\text { s.t. }\left\{\begin{array}{c}
K_{11} \Delta \beta+K_{12} \Delta V=0 \\
Q_{c \text { min }} \leq K_{21} \Delta \beta+K_{22} \Delta V \leq Q_{c \max }+\Delta Q_{c} \\
\Delta \beta_{\text {min }} \leq \Delta \beta \leq \Delta \beta_{\text {max }} \\
\Delta V_{\text {min }} \leq \Delta V \leq \Delta V_{\text {max }}
\end{array}\right.
\end{gathered}
$$

where, $K=\left(\left[\begin{array}{c}L_{l} \\ L_{c} \\ W\end{array}\right]^{-1} \times\left[\begin{array}{c}M_{l} \\ M_{c} \\ D\end{array}\right]-N^{-1} H\right)^{-1} \times\left[\begin{array}{c}L_{l} \\ L_{c} \\ W\end{array}\right]^{-1}$;

$$
K_{11}=M_{l} K ; K_{12}=L_{l} ; K_{21}=M_{c} K ; K_{22}=L_{c} \text {. }
$$

We use the linear programming algorithm to solve (16) and obtain the dual variable $\lambda$ according to the duality principle of the linear programming.

(2) The dual variable is the "marginal cost" of the reactive power compensation device, i.e., the change in the operating cost when the reactive power compensation capacity is increased here. Then we construct the following function for the investment subproblem.

$\min Z$

s.t. $\left\{\begin{array}{c}\sum_{1}^{n_{c}}\left(\lambda_{i}+C_{i}\right) \times \Delta Q_{c i} \leq Z \\ \Delta Q_{c i} \leq \Delta Q_{c i \max }\end{array}\right.$

Where, $\lambda_{i}$ is the dual variable of the operation subproblem;

$C_{i}$ stands for the price of the reactive power compensation capacity at the $\mathrm{i}$-th node unit;

$\Delta Q c_{i}$ is the incremental capacity of the reactive compensation device at the $\mathrm{i}$-th node;

$\Delta Q c_{\text {imax }}$ is the upper limit for the reactive power compensation capacity

$n_{c}$ stands for the number of the reactive compensation nodes.

The objective function of the investment subproblem is the minimum sum of the reactive power equipment investment and the reduced grid loss cost. By solving the investment subproblem, we obtain the new reactive power compensation capacity scheme $Q_{c}^{\prime}$, and then put it back into the operation subproblem to form a new operation subproblem.

By iteration of the operation subproblem and the investment subproblem, we finally obtain the optimal reactive power compensation device allocation plan.

\subsection{Optimization of the generator excitation system adjustment coefficient under multi-mode}

Particle swarm optimization (PSO) is an optimization algorithm inspired from the individual behaviors in the entire system. This algorithm is easy to implement and relies on few empirical parameters, thus it is successfully applied in many optimizations of the power system [19-21]. Recently, PSO has been continuously developed and a number of improved PSO algorithms have been derived, bringing great prospects for the application of PSO. 
From the reactive power compensation device allocation scheme obtained in the first step, we obtain the compensating capacity information of the reactive power at each node as the constants, set the generator excitation system adjustment coefficient value, and calculate the power flow of all the operation modes. Then we calculate the total costs of grid operation by totaling the grid power loss and the reactive power compensation capacity from the flow calculation results under various operation modes, i.e. the objective function value corresponding to the adjustment coefficient. In this paper, we apply the improved particle swarm optimization algorithm to optimize the generator excitation system adjustment coefficient.

\section{EXAMPLE ANALYSIS}

In this paper, we take power grid in some area in Jilin Province as the example. The target grid has $15220 \mathrm{kV}$ substations and $19466 \mathrm{kV}$ substations. We construct the typical operation modes in the planned target year according to the planned grid structure and the load forecasting results. By the above optimization method, we first determine the installed locations and capacities of the reactive power compensation devices and the optimal compensation capacity, and then optimize the generator excitation system adjustment coefficient. The optimized results of the excitation system adjustment coefficients are shown in Table 1.

Table 1. Adjustment coefficient of the generator excitation system in target power system

\begin{tabular}{ccc}
\hline \multirow{2}{*}{ Generator set } & \multicolumn{2}{c}{ Adjustment coefficient/\% } \\
& Before optimization & After optimization \\
\hline G1 & 5.7 & 2.52 \\
G2 & 5.7 & 2.47 \\
G3 & 4.2 & 1.52 \\
G4 & 7.0 & 2.43 \\
G5 & 5.1 & 1.02 \\
\hline
\end{tabular}

As shown in Table 2, before the generator excitation system adjustment coefficient is optimized, the note voltage range is between $0.88 \sim 1.00$ p.u., and the maximum fluctuation range is 0.09 p.u; after optimization, the voltage range is between $0.97 \sim 1.03$ p.u. and the maximum fluctuation range is $0.04 \mathrm{Pu}$. It can be seen that the power grid voltage level is improved significantly due to the installation of power compensation devices and the reasonable optimization of the reactive power adjustment capacity of the generator. With the optimization of the generator excitation system adjustment coefficient, when the load changes, the generator's ability to support the regional grid voltage is utilized more and the voltage fluctuation is greatly reduced.

By calculating the power operating cost and investment cost before and after optimization, we obtain the total costs of power grid operation, as shown in Table 3.

From Table 1, 2 and 3, it can be seen that through the optimization and reasonable setting of the generator excitation system adjustment coefficient, both the reactive power compensation devices and the generator reactive voltage control play their effects.

After optimization, the grid active power loss is decreased, the voltage fluctuation of the grid operation is mitigated, and the comprehensive operating cost of the grid is reduced, bringing significant economic benefits.

We reset the generator excitation system adjustment coefficient in the target power grid according to the results listed in Table 1, and carry out power flow calculation of data under all operation modes in the planned target year to obtain the voltage changes at the nodes before and after the optimization, as shown in Table 2.

Table 2. The comparison of the voltage range before and after adjustment coefficient of the generator excitation system optimization setting in target power system

\begin{tabular}{ccc}
\hline \multirow{2}{*}{ Node No. } & \multicolumn{2}{c}{ Voltage range /p.u. } \\
& Before optimization & After optimization \\
\hline 1 & $0.89 \sim 0.98$ & $0.98 \sim 1.01$ \\
2 & $0.91 \sim 1.00$ & $0.99 \sim 1.03$ \\
3 & $0.93 \sim 1.00$ & $1.01 \sim 1.03$ \\
4 & $0.93 \sim 1.00$ & $1.01 \sim 1.03$ \\
5 & $0.92 \sim 0.99$ & $1.00 \sim 1.03$ \\
6 & $0.88 \sim 0.97$ & $0.97 \sim 1.00$ \\
7 & $0.90 \sim 0.97$ & $0.97 \sim 1.01$ \\
8 & $0.90 \sim 0.98$ & $0.98 \sim 1.01$ \\
9 & $0.89 \sim 0.97$ & $0.97 \sim 1.01$ \\
10 & $0.93 \sim 1.00$ & $1.00 \sim 1.03$ \\
11 & $0.89 \sim 0.98$ & $0.98 \sim 1.01$ \\
12 & $0.91 \sim 0.98$ & $0.99 \sim 1.01$ \\
13 & $0.89 \sim 0.98$ & $1.00 \sim 1.02$ \\
14 & $0.93 \sim 1.00$ & $0.97 \sim 1.01$ \\
\hline
\end{tabular}

Table 3. The benefit analysis of adjustment coefficient of the generator excitation system optimization setting

\begin{tabular}{ccc}
\hline & $\begin{array}{c}\text { Before } \\
\text { optimization }\end{array}$ & $\begin{array}{c}\text { After } \\
\text { optimization }\end{array}$ \\
\hline Grid loss rate, \% & 3.78 & 3.48 \\
grid loss cost, RMB Yuan/h & 2454.71 & 2289.78 \\
investment cost, RMB Yuan /h & & 12.72 \\
Annual yield, RMB 10,000 Yuan & & 133.34 \\
\hline
\end{tabular}

\section{CONCLUSIONS}

In this paper, we propose a reactive power optimal planning model, which takes into account the generator excitation system adjustment coefficient, apply the Benders decomposition algorithm to work out an optimized solution, and based on this, apply the improved PSO to optimize the generator excitation system adjustment coefficient to obtain the optimization scheme for the generator excitation system adjustment coefficient. We apply this method to the actual grid, and the simulation results show that through reasonable setting of the generator excitation system adjustment coefficient, this proposed method can improve the grid voltage level, reduce the active power loss and save investments in reactive power compensation device. 


\section{ACKNOWLEDGMENTS}

Sponsored by the key project of national natural science foundation of China (51437003).

\section{REFERENCES}

[1] Hiscock N., Hazel T.G. (2008). Voltage regulation at sites with distributed generation, IEEE Transactions on Industry Applications, Vol. 44, No. 2, pp. 445-454. DOI: 10.1109/TIA.2008.916749

[2] Davies J.B., Midford L.E. (2000). High side voltage control at Manitoba hydro, Proceedings of 2000 IEEE Power Engineering Society Summer Meeting, Vol. 1, pp. 271-277. DOI: $10.1109 /$ PESS.2000.867595

[3] Zhang Y.J., Ren Z., Li B.F. (2005). Survey on optimal reactive power dispatch of power systems, Power System Technology, Vol. 29, No. 2, pp. 50-56.

[4] Qiu J., Liang C.H. (2004). Strategies and implementation modes of voltage and reactive power control for power plant, Proceedings of the CSUEPSA, Vol. 16, No. 2, pp. 69-72.

[5] An J., Mu G., Zheng T.Y., Wang M.X., Liu B.L., Jiang X. (2013). Optimization strategy for generator excitation System adjustment coefficient in improving voltage level of power system, Automation of Electric Power System, Vol. 37, No. 23, pp. 97-101. DOI: 10.7500/AEPS201301231

[6] Wang M.X., Mu G., An J., Xu X.W., Liu B.L. (2013). Multi-time interval and multi-objective optimization setting of Difference coefficient of generator excitation System, Power System Technology, Vol. 37, No. 11, pp. 178-183.

[7] Wang Q., Zhou S.X., Zhu L.Z. (2003). Improvement of angle stability by advanced high side voltage control regulator, Power System Technology, Vol. 27, no.6, pp. 19-22.

[8] Zhou X.Y., Qiu J.J., Chen X.Q. (2003). Effects of high side voltage control on stabilities for one machine infinite bus, Proceedings of the CSEE, Vol. 23, No. 1, pp. 60-63.

[9] Cheng L., Sun Y.Z., Wu C., Li Y.W. (2007). Effect of load compensation in excitation control on system stabilities, Proceedings of the CSEE, Vol. 27, No. 9, pp. 32-37.

[10] Noguchi S., Shimomura M. (2006). Improvement to an advanced high side voltage control, IEEE Transactions on Power Systems, Vol. 21, No. 2, pp. 683-692.
[11] Wang Q., Zhu L.Z., An N., Zhou S.X. (2004). Improvement of voltage stability with an advanced high side voltage control regulator, Journal of Tsinghua Uni: Sci \& Tech Edition, Vol. 44, No. 1, pp. 102-105.

[12] Liu M.B., Yang Y. (2005). Optimal reactive power planning incorporating steady state voltage stability constraints, Automation of Electric Power Systems. vol. 29, No.5, pp. 21-25.

[13] $\mathrm{Hu}$ C.E., Yang R.P. (2005). Optimal planning for reactive power with voltage stability, RELAY, Vol. 33, No. 4, pp. 22-30.

[14] Li L.C., Wang J.Y., Chen L.Y., Song W.N. (1999). Optimal reactive power planning of electrical power system, Proceedings of the CSEE, Vol. 19, No. 2, pp. 66-69.

[15] Wang C.N., Tang X.L., Yu Y.X. (1998). Optimal Reactive Power Planning in Urban High and Middle Voltage Power Distribution Network, Power System Technology, Vol. 22, No. 8, pp. 23-26.

[16] Jiang B.Y., Xie J., Du D.M. (1993). Application of the generalized Benders decomposition to reactive capacity allocation planning, Journal of North China Institute of Electric Power, Vol.4, No. 2, pp. 29-37.

[17] Wang C.S., Tang X.L., Yu Y.X., Zhang Y. (1998). Optimal reactive power planning using heuristic method and Benders decomposition technique, Automation of Electric Power System, Vol. 22, No. 11, pp.14-17.

[18] Chen H.Y., Chen J.F., Duan X.Z. (2008). Reactive power optimization in distribution system with wind power generators, Proceedings of the CSEE, Vol. 28, No. 7, pp. 40-45.

[19] Zhang T.C., Geng G.F. (1998). Reactive power optimization for medium voltage distribution network based on improved particle swarm optimization, Power System Technology, Vol. 22, No. 8, pp. 23-26.

[20] Fan C.L., Xing Q.H., Li X., Wang Z.J. (1998). Improved particle swarm optimization algorithm with reverse forecast and repulsion, Control and Decision. vol. 22, No. 8, pp. 23-26.

[21] Hou Y.H., Lu L.J., Xiong X.G., Shi C.J., Wu Y.W. (2004). Enhanced Particle Swarm Optimization algorithm and its Application on Economic Dispatch of Power System, Proceedings of the CSEE, Vol. 24, No. 7, pp. 95-100. 